Research Paper

\title{
Downregulation of DACT-2 by Promoter Methylation and its Clinicopathological Significance in Prostate Cancer
}

\author{
Shibao Li1 ${ }^{12^{*}}$, Lingyu Yin ${ }^{1,2^{*}}$, Kai Huang ${ }^{3^{*}}$, Yao zhao ${ }^{1}$, Haoliang Zhang ${ }^{1,2}$, Chenchen Cai ${ }^{1}$, Yinhai Xu ${ }^{2}$, \\ Lingyan Huang ${ }^{1}$, Xiaozhou Wang ${ }^{4}$, Ting Lan ${ }^{1}$, Hongchun $\mathrm{Li}^{1,2}{ }^{\bowtie}$, Ping Ma ${ }^{1,2} \bowtie$ \\ 1. School of Medical Technology, Xuzhou Medical University, Xuzhou 221004, China; \\ 2. Department of Laboratory Medicine, Affiliated Hospital of Xuzhou Medical University, Xuzhou 221002, China; \\ 3. Department of Urology, Northern Jiangsu People's hospital, Yangzhou 225001 China; \\ 4. The center of functional experiment, Xuzhou Medical University, Xuzhou Jiangsu 221004, China. \\ *Equal contributors \\ $\bowtie$ Corresponding authors: Pro Hongchun Li (13775891123@163.com) , Ping Ma (pingm62@aliyun.com); Phone: +86-516-83353651
}

(c) Ivyspring International Publisher. This is an open access article distributed under the terms of the Creative Commons Attribution (CC BY-NC) license (https://creativecommons.org/licenses/by-nc/4.0/). See http://ivyspring.com/terms for full terms and conditions.

Received: 2018.07.17; Accepted: 2019.01.16; Published: 2019.04.20

\begin{abstract}
Backgrounds: Dapper homolog (DACT) 2, a member of DACT gene family, is frequently down-regulated in various malignancies and linked to tumor progression. However, the regulatory mechanism of DACT-2 expression and its biological role in human prostate cancer $(\mathrm{PCa})$ remains elusive. Here, we investigated the expression and an epigenetic change of DACT-2 in prostate cancer, and determined if these findings were correlated with clinicopathologic characteristics of PCa.

Methods: The expression profile of DACT-2 of was detected by qRT-PCR, Western blotting, and immunohistochemistry in four prostate cell lines (RWPE-1, LNCaP, PC-3 and DU145), 56 cases of frozen prostate tissues (forty-seven primary prostate carcinomas, nine paired noncancerous and cancerous prostate tissues) and a tissue microarray sets including 100 paraffin-embedded prostate samples ( 3 normal tissues, 2 cases of adjacent tissues and 95 cases of cancer). Subsequently, the regulatory mechanism of DACT-2 down-regulation was investigated through methylation-specific PCR (MSP) and bisulfite sequencing (BSP). The role of DACT-2 in prostate cancer cell migration and invasion was respectively examined by wound healing and transwell assay. After 5-aza-2'-deoxycytidine treatment of prostate cancer cells, qRT-PCR was used to detect whether the expression of DACT-2 gene mRNA in the cells recovered.

Results: Immunohistochemical results shown that the DACT-2 protein was strongly $(3+)$ expressed in the cytoplasm of all 5 noncancerous tissues and 12.7\% (12/95) prostate cancer (PCa) tissues. Whereas $68.4 \%$ (65/95) PCa samples and 18.9\% (18/95) PCa tissues respectively displayed weakly $(1+)$ expressed and moderately $(2+)$ expressed. In addition, DACT-2 expression was negatively associated with Gleason score in tumor specimens $(p=0.029)$. What's more, down-regulation and promoter methylation of DACT-2 were observed in 68.1\% (32/47) frozen PCa tissues and all three prostate cancer cell lines. And, the expression of DACT-2 mRNA was restored by the treatment of demethylated drug 5-aza-2'-deoxycytidine in all prostate cancer lines. Prostate cancer cells invasion and migration were significantly suppressed by ectopic expression of DACT-2 in vitro.

Conclusions: Our study provides evidence that DACT-2 may be a useful biomarker for distinguishing prostate tumor tissues from non-cancerous samples and a potential target for epigenetic silencing in primary prostate Cancer.
\end{abstract}

Key words: prostate cancer, DACT-2, methylation, Gleason score, TNM staging

\section{Background}

Currently, prostate cancer $(\mathrm{PCa})$ is still one of the most difficult problems that can hardly be overcome worldwide and the mortality rate of $\mathrm{PCa}$ is only inferior to that of skin cancer in men ${ }^{[1,2]}$. Meanwhile, 
the burden of this disease has been increasing, that is, the incidence of the disease will increase to 1.7 million by 2030 with the expansion and aging of the global population as well as lifestyle ${ }^{[3,4]}$. Most PCa arises from intraepithelial neoplasia that requires years or even decades to develop malignant neoplasms ${ }^{[5]}$. The initiation and progression of PCa is a complicated and incompletely understood process driven by genetic and epigenetic alterations ${ }^{[6,7]}$. Wherein, the inactivation of tumor suppressor gene via promoter methylation has been identified to be the corresponding molecular events of PCa carcinogenesis[8]. Therefore, identifying new molecular modification related to the development of this devastating disease would aid the management and personalized treatment of patients with PCa.

Human DACT-2 is a novel signal transduction molecule discovered in recent years, which was expressed in placenta, genitourinary system tumor and normal colonic mucosa and colorectal cancer tissues ${ }^{[9]}$. Accumulated evidence have demonstrated that DACT-2 is down-regulated through its promoter methylation in multiple solid tumors ${ }^{[10-13]}$, implying that DACT-2 may be a potential tumor suppressor gene involved in the occurrence and development of tumors. However, the expression profile and its epigenetic change of DACT-2 in PCa remain unknown.

Here, we mainly investigated the expression and its regulatory mechanism of DACT-2 in prostate cell lines and prostate tissue, and analyzed the relationship between DACT-2 alterations and clinical characteristics of these patients. Besides, we also investigated its biological function in PCa cells. This study will help to gain a better understanding of epigenetic changes in $\mathrm{PCa}$, which may be appropriate for the diagnosis, prognosis and treatment of prostate cancer.

\section{Materials and Methods}

\section{Cell culture}

Four prostate cell lines (RWPE-1, LNCaP, PC-3 and DU145) were all enrolled in the current study. All of the above cell lines were cultured in the mixture of RPMI 1640 medium (HyClone, Logan, UT), 10\% fetal bovine serum and penicillin $(100 \mathrm{U} / \mathrm{ml})$ and streptomycin $(100 \mathrm{U} / \mathrm{ml})$ at $37^{\circ} \mathrm{C}$ with $5 \% \mathrm{CO}_{2}$.

\section{Clinical samples}

All tissue specimens used in this study were from the affiliated hospital of Xuzhou Medical University and Northern Jiangsu People's hospital (January 2012 to December 2016), a total of 156 tissue samples (aged 56-96 years, the median age of 71 years) containing 100 paraffin-embedded prostate tissues comprising a tissue microarray sets analyzed by immunohistochemistry ( 3 normal prostate tissues, 2 paraffin-embedded adjacent tissues and 95 paraffinembedded tumor tissues) and 56 frozen tissue samples used (47 PCa and 9 adjacent non-cancerous tissues). Histological evidence of all cancerous samples was verified to contain more than $80 \%$ of tumor cells. According to the 1997 TNM classification system, the formalin fixation specimens were evaluated for pathological stage. Written consent is obtained from all subjects, and the research program is approved by the affiliated hospital of Xuzhou Medical University Ethics Committee and Northern Jiangsu People's Hospital Ethics Committee.

\section{RNA isolation and semi-quantitative RT-PCR}

Total RNA of prostate cell lines were extracted using Trizol reagent (TaKaRa, China) as described in the manufacturer's instructions. Spectrophotometer (ne Drop, OD-1000+) was employed to detect the concentration of RNA, which was reverse transcribed to cDNA using the PrimScript (TM) RT kit based on the protocol (TaKaRa, China). Using the above cDNA as the template, the levels of GAPDH served as an internal control and DACT-2 mRNA were checked by RT-PCR Roche LightCycler 480 II detection system. The real-time PCR reaction system was $20 \mu \mathrm{l}$, and the cycling condition is $95^{\circ} \mathrm{C} 5 \mathrm{~min},\left(95^{\circ} \mathrm{C} 30 \mathrm{~s}, 58^{\circ} \mathrm{C} 30 \mathrm{~s}\right.$, $\left.72^{\circ} \mathrm{C} 30 \mathrm{~s}\right) \times 38,72^{\circ} \mathrm{C} 7 \mathrm{~min}$. The relative expression level of DACT-2 mRNA was calculated by $2-\Delta \triangle C T$ method. It is believed that increasing or decreasing the level of mRNA by at least two fold is significant. The DACT-2 PCR primer sequences were as follows: 5'-GGC TGA GAC AAC AGG ACA TCG-3' (F) and 5'-GAC CGT CGC TCA TCT CGT AAAA-3' (R); GAPDH PCR primer sequences: 5'-GAGAAGGCTGG GGCTCATTT-3' (F) and 5'-AGTGATGGC ATGGACT GTGG-3' (R). The experiment was repeated three times under the same conditions.

\section{Western blot analysis}

Radioimmunoprecipitation assay (RIPA) buffer was used to abstract the total protein of the prostate cell lines, and protein concentration was measured by the BCA Protein Reagent Kit (Beyotime, China) as shown in the instructions. The prepared proteins were then separated by SDS-PAGE with $10 \%$ gel and electroblotted onto a polyvinylidene fluoride membrane (PVDF membrane). The proteins were blocked at room temperature for 2 hours at 5\% skim milk with TBS. After that the following antibodies were applied to incubate overnight on the membrane, including: rabbit anti-DACT-2 (1:200, OriGene Tech, USA) and rabbit anti- beta-actin (1:1000). The membrane was then incubated goat polyclonal secondary antibody 
with fluorescenceto rabbit IgG (1: 5000, Abcam, CA) for 2 hours. Finally, the protein is scanned with Odyssey Infrared Imaging (Odyssey, LI-COR, USA).

\section{Immunohistochemistry}

Immunohistochemistry (IHC) was performed in tissue microarray sets including 3 normal prostate tissues, 2 cases of adjacent tissues and 95 cases of PCa. DACT-2 antibody (OriGene Tech, USA) was diluted to $1 / 2000$ dilution, and the experiment was carried out following the instructions of immunohistochemistry kit. Next, the sections were stained with hematoxylin after incubation of the DAB developer for several minutes, and finally taken by Olympus BX53 microscopy and photographed with Cellsens Entry software (Olympus). The expression level of DACT-2 was assessed by the calculation of the percentage and intensity of the positive tumor cells. The expression intensity of DACT-2 protein in tissue was recorded by $0,1,2$ and 3 , respectively, representing negative, weakly positive, moderately positive and strong positive. The degree of expression of DACT-2 is defined as a percentage of the positive staining area associated with the entire tissue region. The criteria for DACT-2 expression are as follows: $0 \%$ $=0,1-10 \%=1,11-50 \%=2,51-80 \%=3,81-100 \%=4$. The final immunoreactive fraction (0 to 12 ) was determined by multiplying the intensity score by the degree of staining cell score. Finally, when the total score was below 2, the classification of the expression of DACT-2 was considered as negative/weak; When the total score was between 3 and 6 , it was classified as moderate expression; When the total score was $\geq 7$, it was classified as strong moderate expression ${ }^{[14]}$.

\section{Methylation-specific PCR}

On the basis of the manufacturer's instructions, DNA Kit was utilized to obtain genomic DNA in prostate tissues and cell lines, and the bisulfite modification assay was performed by the EZ DNA Methylation-Gold Kit (ZYMO Research Co, Orange, CA). To detect the methylation of DACT-2 gene in prostate tissues and cell lines, methylated primers and unmethylated primers were applied to amplify the modified DNA. The methylated primers included forward primer: 5'-GCG CGT GTA GATTTC GTT TTT CGC-3' and reverse primer: 5'-AAC CCC ACG AAC GACGCCG-3'. The unmethylated primers consisted of forward primer: $5^{\prime}$-TTG GGG TGT GTG TAG ATT TTGTTT TTTGT-3' and reverse primer: $5^{\prime}$-CCC AAA CCC CAC AAA CAACAC CA-3'. The amplification system used in this experiment was $20 \mu \mathrm{l}$, consisting of $10 \mu \mathrm{l}$ of Taq PCR MasterMix, $7 \mu \mathrm{l}$ of nuclease-free water, $1 \mu l$ of each upstream and downstream primers and $1 \mu \mathrm{l}$ of modified DNA. The amplification conditions involved in this reaction are as follows: $95^{\circ} \mathrm{C} 5 \mathrm{~min},\left(95^{\circ} \mathrm{C} 30 \mathrm{~s}, 58^{\circ} \mathrm{C} 30 \mathrm{~s}, 72^{\circ} \mathrm{C} 30 \mathrm{~s}\right)$ $\times 38,72^{\circ} \mathrm{C} 7 \mathrm{~min}$. The amplified product was separated on a $3 \%$ agarose gel and visualized under UV illumination.

\section{Bisulfite genomic sequencing}

Bisulfite genomic sequencing (BSP) was carried out as previously described. Briefly, the modified DNA was amplified via PCR using the BSP primer as following: 5'-GGG GGA GGT YGY GGT GAT TT-3' (F) and 5'-ACC TAC RAC RAT CCC AAC CC-3' (R). Then, the amplifications were purified, subcloned into the pMD19-T vector (TaKaRa) and transformed into E. coli (DH5-alpha) according to the manufacturer's protocol. To investigate the methylation status of the DACT-2 promoter, six isolated colonies, grown on LB agar plates containing kanamycin with X-gal/IPTG, were picked, sequenced and analyzed with an $\mathrm{ABI}$ 3730 DNA Sequencer (Applied Biosystems ${ }^{\circledR}$ ).

\section{5-aza-2'-deoxycytidine treatment}

Prostate cell lines were divided into low density ( $30 \%$ confluence) in a 6-well plate 36 hours prior to drug treatment. The cells were incubated for $24 \mathrm{~h}$ and $48 \mathrm{~h}$ in growth medium with or without demethylating agent 5 -aza-2'-deoxycytidine $(5 \mu \mathrm{mol} / \mathrm{L}$, SigmaAldrich, USA). The growth medium was exchanged every 24 hours. After the above process, the RNA was extracted according to the previous method.

\section{Scratch migration assay and Invasion assay}

To assess DACT-2 function, we obtained the PC-3 derived cell line stably overexpressing DACT-2 by transfection of DACT-2 lentiviral vectors, which were packed using the DACT-2 pLVX-IRES-ZsGreen1 plasmid (YouBia, China) according to the manufacturer's protocol. PC-3 cells transfected with the empty lentiviral vector were used as controls.

Migration: $5 \times 10^{5}$ DACT-2 control and re-expressed PC-3 cells were suspended in $200 \mu \mathrm{l}$ serum-free RPMI 1640 media and added to the upper chamber of $8.0 \mu \mathrm{m}$ pore size transwell apparatus (COSTAR transwell, Corning Incorporated, MA, USA). Cells that migrated to the lower surface of the membrane were stained with crystal violet and counted in five independent high-power fields $(\times 100)$ after incubating for 24 hours.

Matrigel invasion assay: $5 \times 10^{5}$ cells were seeded into the upper compartment of the insert in Matrigel (BD Biosciences, Woburn, Mass) in serum free growth medium. Then, the upper chamber was placed in a 24-well culture dish containing $600 \mu l$ of complete growth medium. After 48 hours of incubation at $37^{\circ} \mathrm{C}$, the cells in the upper chamber were subsequently removed with a cotton swab and 
then stained with a solution containing $0.1 \%$ crystal violet and $4 \%$ formaldehyde. Count the number of cells immobilized on the basement membrane of the insert.

For the scraping migration assay, cells were grown in 6-well plates until confluent. The monolayer was scraped with a sterile $20 \mu \mathrm{l}$ pipette tip to create a bare area of constant width. Wound closure was monitored and photographed before and 24 hours after wounding.

\section{Statistics}

SPSS19.0 software was conducted for statistical analysis. The mRNA expression of DACT-2 gene was expressed as the mean \pm standard deviation and the means were compared using the Student's $t$ test. The association between DACT-2 protein expression and clinicopathological parameters was analyzed by Chi-square test. $P<0.05$ for the difference was statistically significant.

\section{Results}

\section{Down-regulation of DACT-2 in prostate cancer}

Real-time PCR was performed to detect the expression of DACT-2 transcript in a panel of human prostate cell lines (RWPE-1, LNCaP, PC-3, and DU1 45). Compared with the nonmalignant RWPE-1 cells, the mRNA level of DACT-2 was dropped sharply in all three PCa cell lines (Figure 1A). The similar results were also found in the DACT-2 protein levels by western blot analyses (Figure 1B).

We next detected DACT-2 protein level by immunohistochemistry in 100 prostate tissue specimens, including 3 normal tissues, 2 adjacent tissues, and 95 primary prostate tumor tissues. Strong immunostaining of the DACT-2 protein was seen in the cytoplasm of normal tissues and adjacent tissues (Figure 2A\&2B). The DACT-2 was negatively or weakly expressed in 65 of $95(68.4 \%)$, moderately expressed in 18 of $95(18.9 \%)$ and strongly expressed in 12 of $95(12.7 \%)$ tumor samples of PCa (Figure 2C, 2D). Intriguingly, DACT-2 down-regulation was significantly more common in the high Gleason score group $(40 / 50)$ than that the low Gleason score group $(25 / 45)(p=0.029)$. No correlation between the DACT-2 down-regulation and median age $(p=0.881)$, TNM stage $(p=0.461)$, PSA serum concentration $(p=0.518)$ or prostate volume $(p=0.876)$ was observed in prostate carcinoma samples (Table I). These above evidence implied that DACT-2 may act as tumor suppressor genes in prostate cancer.

Table I. The relationship between the expression of DACT-2 and clinicopathological parameters

\begin{tabular}{|c|c|c|c|c|c|}
\hline \multirow[b]{2}{*}{ Parameters } & \multirow[b]{2}{*}{ No. } & \multicolumn{3}{|c|}{ The Expression of DACT-2 Protein } & \multirow[b]{2}{*}{$P$} \\
\hline & & Negative/Weak & Moderate & Strong & \\
\hline & 95 & 65 & 18 & 12 & \\
\hline Age (years) & & & & & 0.881 \\
\hline$<=70$ & 46 & 32 & 9 & 5 & \\
\hline$>70$ & 49 & 33 & 9 & 7 & \\
\hline TNM & & & & & 0.461 \\
\hline T1- T2 & 42 & 28 & 10 & 4 & \\
\hline T3- T4 & 53 & 37 & 8 & 8 & \\
\hline Gleason score & & & & & 0.029 \\
\hline $6-7$ & 45 & 25 & 11 & 9 & \\
\hline $8-10$ & 50 & 40 & 7 & 3 & \\
\hline PSA (ng/ml) & & & & & 0.518 \\
\hline$<=10$ & 28 & 19 & 4 & 5 & \\
\hline$>10$ & 67 & 46 & 14 & 7 & \\
\hline Prostate volume (ml) & & & & & 0.876 \\
\hline$<=50$ & 26 & 17 & 5 & 4 & \\
\hline$>50$ & 69 & 48 & 13 & 8 & \\
\hline
\end{tabular}

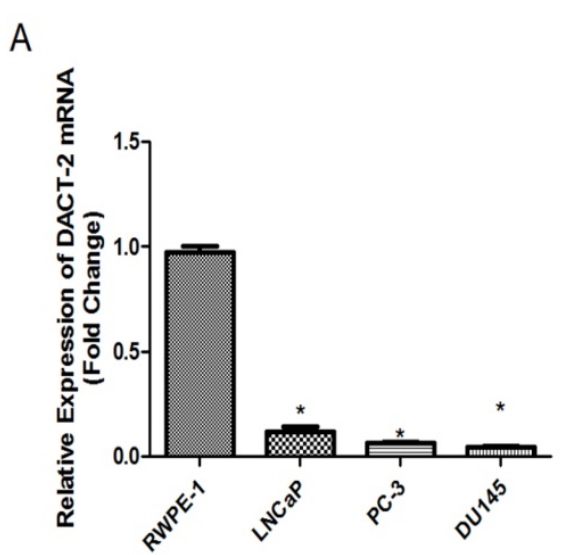

B

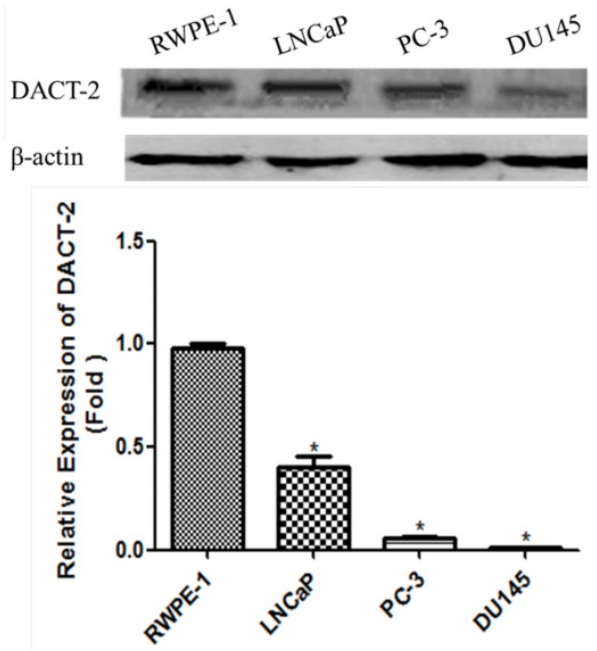

Figure 1. qRT-PCR and Western blot analysis of DACT-2 expression in prostate cell lines. A, qRT-PCR analysis of DACT-2 mRNA expression in four prostate cell lines and $\beta$-actin as an internal reference. B, DACT-2 protein ( $83 \mathrm{kDa}$ band ) expression was detected by Western blot analysis and $\beta$-actin ( $43 \mathrm{kDa}$ band ) was used as a control. 

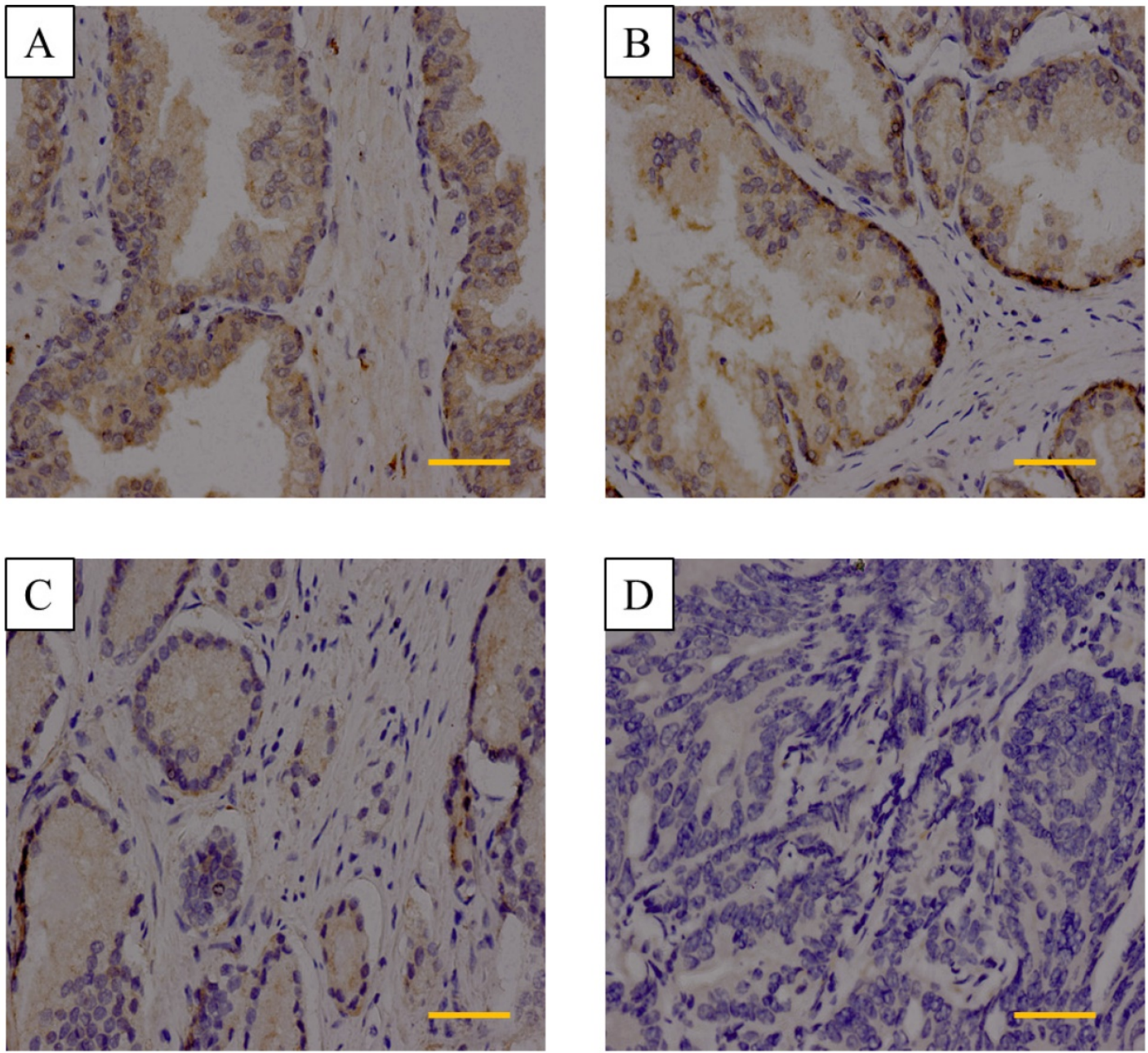

Figure 2. Immunohistochemistry analysis of DACT-2 expression in prostate tissue specimens. A\&B, is representative of normal prostate tissue and adjacent tissues, respectively. C, Weak cytoplasmic expression of DACT-2 in the prostate carcinoma tissue sample 24(Gleason score $=3+4$ ). D, Complete loss of DACT-2 expression in the prostate carcinoma sample 63 (Gleason score $=4+5)$ (scale bar $=50 \mu \mathrm{m}) .(400 \times$ original magnification).

\section{Down-regulation of DACT-2 is associated with hypermethylation of the CpG island in its promoter}

In order to clarify the mechanism of DACT-2 gene silencing in PCa cell lines, we analyzed the DAC-2 gene 5 ' regulatory region. There was a CpG islands in the transcription initiation site of DACT-2. Then, we analyzed a 255 bp segment of this region (-114 to +141 bp; TSS, +1 bp), which includes 35 CpG sites and spans the core promoter and part of exon 1 , using bisulfite genomic sequencing (BSP) (Figure 3B). Only $2.4 \%$ hypermethylated CpG sites was detected in the RWPE-1 nonmalignant cell line, whereas more than $60 \%$ hypermethylation could be observed in all three tumor cell lines (LNCaP, PC-3, and DU145) (Figure 3C).

To optimize the detecting method of DACT-2 hypermethylation, we designed methylated specific primers and unmethylated specific primers according to BSP results, and analyzed the methylation status of
DACT-2 gene promoter using methylation-specific PCR (MSP) in four prostate cell lines. Similar to the BSP results, methylation of DACT-2 was observed in all three prostate cancer cell lines, whereas no methylated DNA was detected in the normal prostate epithelial cell line RWPE-1, suggesting that DACT-2 gene silencing in prostate cancer cell lines may be closely related to the hypermethylation of DACT-2 promoter (Figure 3A).

To further figure out the relationship between DACT-2 expression and promoter methylation, all cell lines were treated by 5-aza-2'-deoxycytidine (5-Aza, 5 $\mu \mathrm{mol} / \mathrm{L})$. After 24 hours and 48 hours of treatment, the mRNA levels of DACT-2 were significantly increased in LNCaP, PC-3, DU145 compared to untreated cells, whereas the mRNA level of DACT-2 in RWPE-1 was almost unchanged (Figure 4), through DNA hypermethylation. These results suggested that down-regulation of DACT-2 expression was caused by hypermethylation of its promoter in prostate cancer cell lines. 

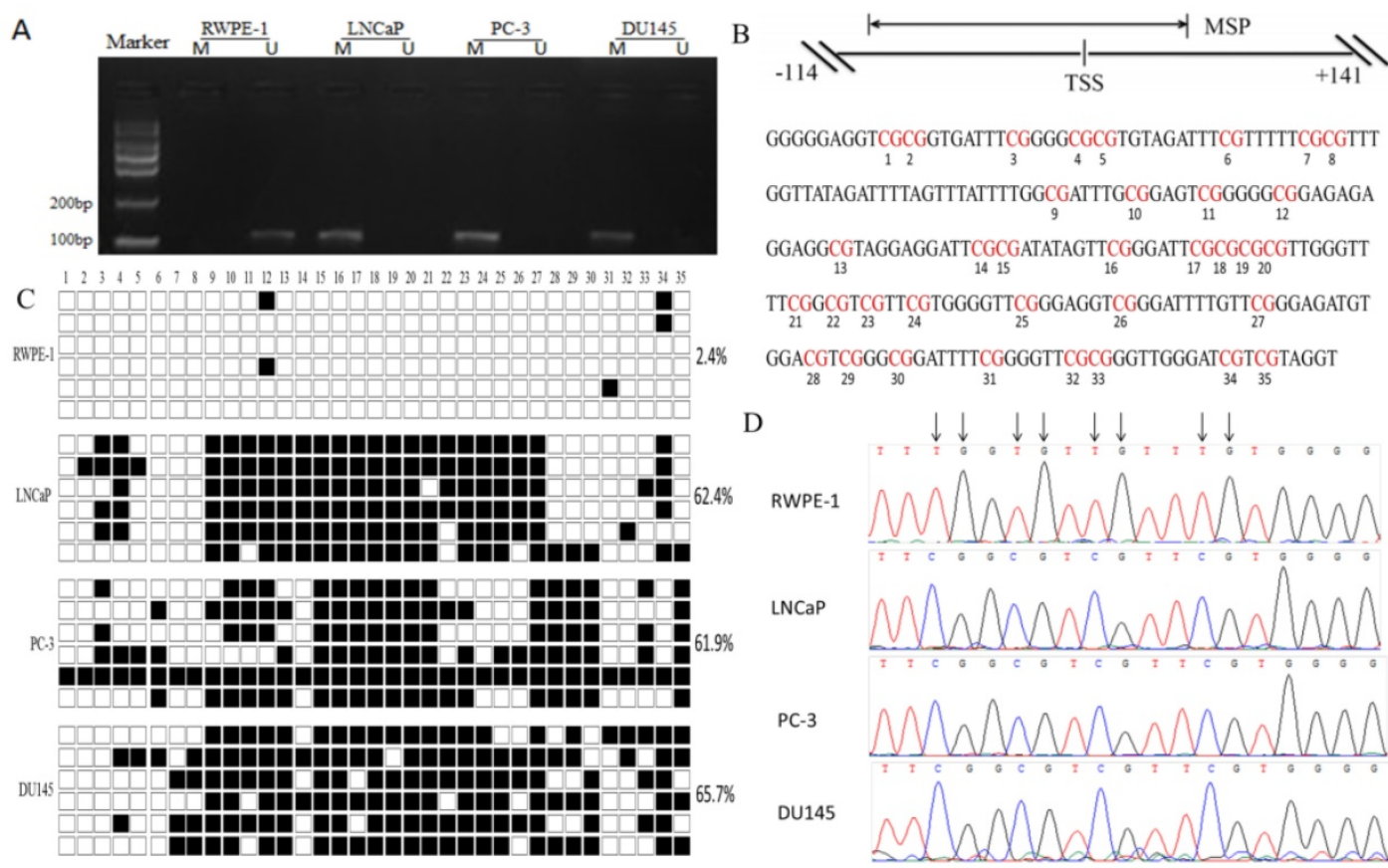

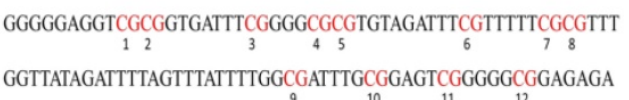

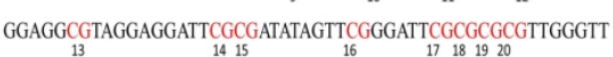

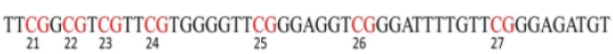

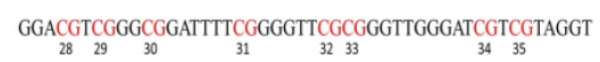

D

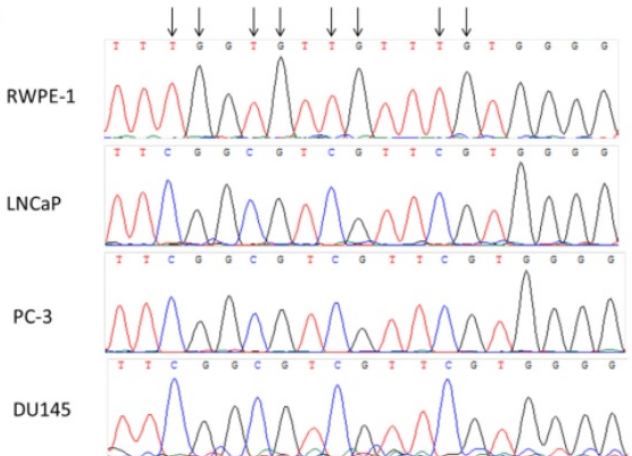

Figure 3. DNA hypermethylation is a major mechanism of the downregulation of DACT-2 in prostate cancer cell lines. A. Methylation status of the DACT-2 gene promoter in prostate cell lines. The DACT-2 methylation status was analyzed by MSP-PCR. All prostate cancer cell lines showed complete methylation of the DACT-2 gene. Unmethylated DACT-2 gene was detected in RWPE-1 cells. The lanes labeled "M"and"U" represent products that were amplified with primers that recognize the methylated and unmethylated sequences, respectively. B, Schematic depiction of DACT-2 promoter-associated CpG island. It spans the region from -114 to +141 with respect to the TSS $(+1)$. Regions of MSP and bisulfite genomic sequencing (Bis-DACT-2) are shown. Bis-DACT-2 has 255 bP and contains 35 CpG dinucleotides. C, Methylation pattern of the Bis-DACT-2 region of the DACT-2 CpG island in four cell lines. Methylated and unmethylated CpG sites were shown as solid squares and open squares, respectively ( $\square$, methylated cytokines; $\square$, unmethylated cytosines). Cell-line names and the percentage of methylation for the corresponding cell line are indicated on the left and right sides, respectively. D, Representative chromatograms of $C_{p} G$ sites 15 to 18 obtained from bisulfite sequencing of the DACT-2 fragment. Arrows indicate positions of CpG dinucleotides.

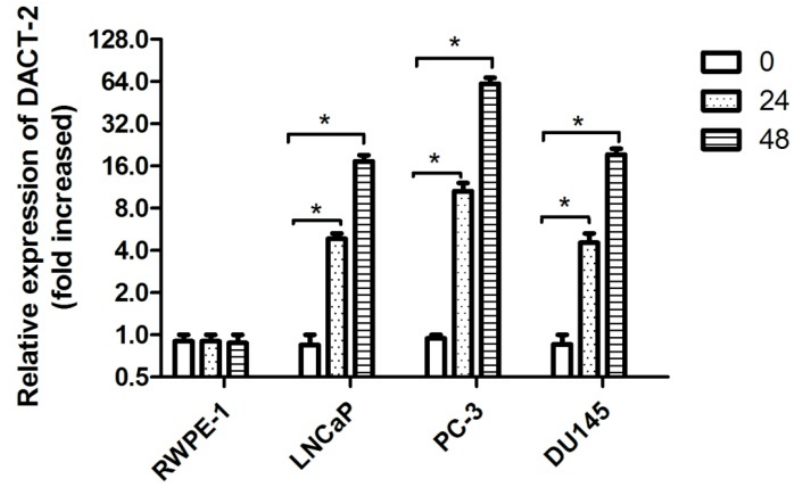

Figure 4. DACT-2 mRNA expression analysis by $q R T-P C R$ in 4 prostate cells lines following 5-aza-2'-deoxycytidine (5 $\mu \mathrm{mol} / \mathrm{L})$ treatment for $0, \mathbf{2 4 h r}$ and $48 \mathrm{hr}$. The DACT-2 gene expression level of the cell line treated with 5-aza-2'-deoxycytidine showed a relative fold change $(0$ hours, defined as 1.0) compared to untreated cells. * Indicates a statistically significant difference of $P<0.05$ compared with the 0 hour value.

\section{Correlation analysis of DACT-2 protein expression and methylation in primary prostate cancer}

To further ascertain whether the downregulation of DACT-2 was also introduced by promoter hypermethylation in primary prostate tumors, we analyzed the methylation status and the protein expression of DACT-2 in 47 frozen prostate tumor tissues and 9 paired noncancerous tissues respectively using MSP and Western blotting. Methylated DACT-2 was detected in all tumor tissues, whereas no methylated DACT-2 was observed in all matched non-cancerous tissues (Figure 5). Of these 47 methylated tumor tissues, DACT-2 protein expression was markedly downregulated or lost in 32 samples (Figure 6). The correlation between DACT-2 expression and hypermethylation of the $\mathrm{CpG}$ island was significant $(p=0.001)$, implying that the hypermethylation of DACT-2 was the major regulatory mechanism of DACT-2 down-regulation in prostate tumor $(p<0.01)$.

\section{DACT-2 suppresses cell migration and invasion in prostate cancer cells}

To evaluate the effects of DACT-2 on cell migration and invasion, the transwell and wound healing assays were carried out in PC-3 cells. Western blotting analysis demonstrated that DACT-2 was over-expressed in PC-3 cells transfected with DACT-2 lentiviral vector (Figure 7A). Under the transwell assay, the number of migratory cells was $70 \pm 7.20 \mathrm{vs}$. $18.8 \pm 2.72$ for PC-3 cells before and after restoration of DACT-2 expression. The cell number was reduced significantly after re-expression of DACT-2 in PC-3 $(p<0.001$, Figure 7B). These results suggested that 
DACT-2 suppresses prostate cancer cell migration. The number of invasive cells was $42 \pm 2.65$ vs. $19 \pm$ 3.46 for PC-3 cells before and after restoration of DACT2 expression. The invasive cell number was reduced significantly after re-expression of DACT2 in PC-3 cells $(p<0.001$, Figure 7C). These results suggest that DACT-2 can suppress prostate cancer cell migration and invasion. The cell migration ability was evaluated by the wound healing assay as well in PC-3 cells. As shown in Figure 7D, cell migration was sharply suppressed after re-expression of DACT-2 in PC-3 cells. The scratch fusion was $77 \pm 4.50$ vs. $23.8 \pm$ 3.70 for PC-3 cells before and after restoration of DACT-2 expression $(p<0.001$, Figure 7D). Taken together, the above results demonstrated that DACT-2 suppressed prostate cancer cell invasion and migration.

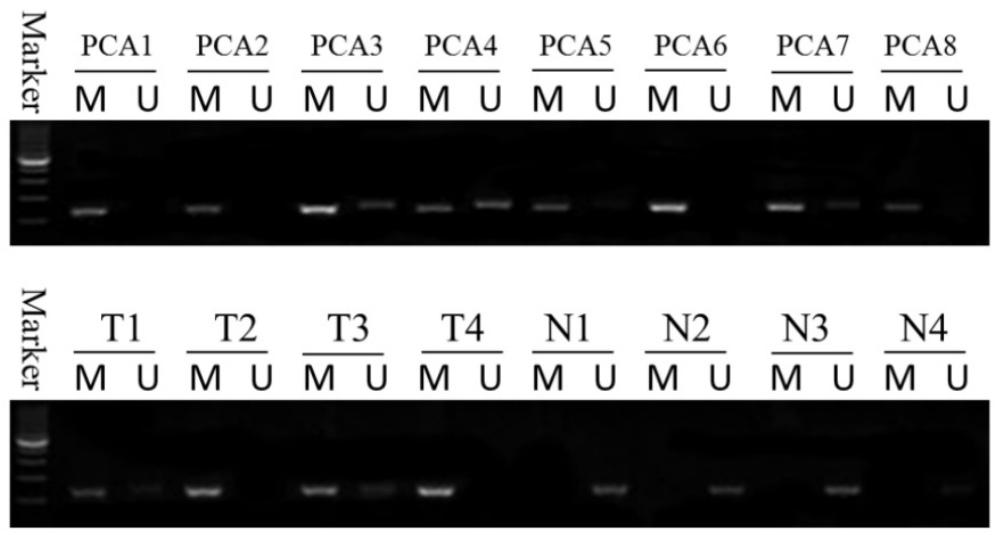

Figure 5. Methylation status of the DACT-2 gene promoter in prostate tissue. DACT-2 methylation status was determined by MSP-PCR. All of the prostate cancer tissues exhibit complete methylation of the DACT-2 gene (PCA1-PCA8). The unmethylated alleles were detected in adjacent noncancerous tissues (T1-T4: prostate cancer tissue; N1-N4: adjacent noncancerous tissues). Lanes labeled " $M$ " and " $U$ " denote products amplified by primers recognizing methylated and unmethylated sequences, respectively.
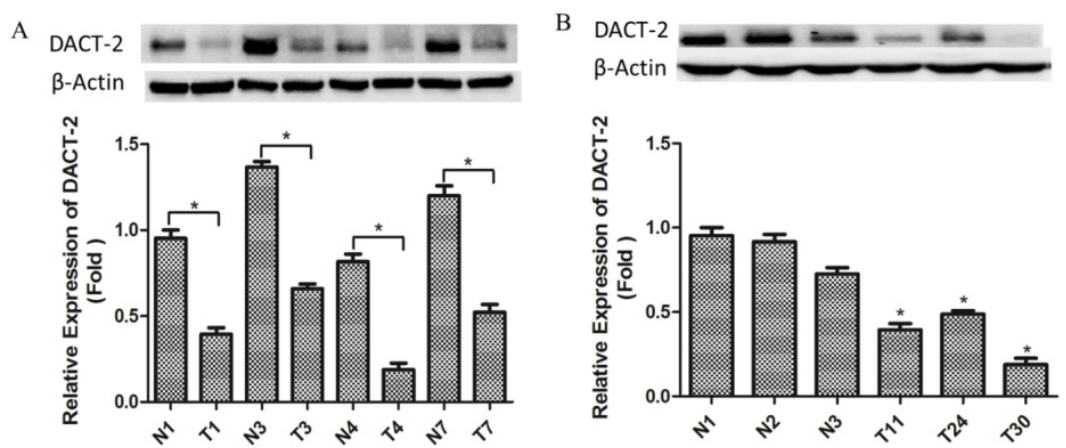

Figure 6. Western blotting analysis of DACT-2 protein expression in clinical prostate specimens. A, DACT-2 protein ( $83 \mathrm{kDa}$ band ) levels in representative resected fresh prostate cancer tissues and matched non-tumor tissues were analyzed by western blotting and normalized to $\beta$-actin expression ( $43 \mathrm{kDa}$ band ). Expression was further normalized to the expression level observed in the first noncancerous specimen. B, DACT-2 protein levels ( $83 \mathrm{kDa}$ band) in representative fresh prostate cancer tissues and noncancerous specimen were analyzed by western blotting and normalized to $\beta$-actin ( $43 \mathrm{kDa}$ band). Protein levels were further normalized to the expression level observed in the first noncancerous specimen. Representative results from triplicate experiments are shown as mean $\pm S D(n=3)$. $* P<0.05$, vs. the respective noncancerous tissue. $N=$ non-tumor tissue; $\mathrm{T}=$ tumor tissue.

\section{Discussion}

This study systematically explored the expression profile of DACT-2 and it's clinical relevance in prostate cell lines and tissues, and uated the relationship between DACT-2 frequently silenced by its promoter hypermethylation prostate cancer, implying that the transcriptional silencing of DACT-2 may be one of the essential

Accumulated evidence has demonstrated that DACT-2 is not only related to development processes of organisms [15], but also plays a vital role in recent report has confirmed that DACT-2 expression was down-regulated in multiple colon cancer cell lines but emerged strong expression in normal colonic tissue, suggesting that DACT2- may act as an effective tumor suppressor in colon cancer[10]. In addition, previous studies also revealed that the down-regulation of DACT-2 protein expression occurred in many other tumors, including human esophageal cancer, thyroid cancer and gastric cancer[11-13]. Consistent with previous studies, the downregulation of DACT-2 expression was also observed in 32 of $47(68.1 \%)$ prostate cancer tissues with various histological stages and in all 3 PCa cell lines. Moreover, immunohistochemical results showed that the DACT-2 was highly expressed in normal prostate tissues and adjacent tissues, whereas $68.4 \%$ of the tumor tissues were significantly reduced or lost, implying that the downregulation of DACT-2 may be a useful marker of distinguishing between cancerous and non-cancerous prostate tissues. What's more, the expression of DACT-2 was negatively correlated with increased Gleason score in patients with $\mathrm{PCa}$, and the ectopic expression of DACT-2 significantly decreased PC-3 cell migratory and invasive capability in vitro. The above evidence suggested a potentially suppressive role of DACT-2 in prostate tumor progression.

Promoter hypermethylation was an important mechanism of critical genes silencing. Previous studies have demonstrated DACT-2 gene silenced by methylation in many tumors, including 
colon cancer, esophageal cancer, thyroid cancer and gastric cancer [10-13]. Similarly, methylated DACT-2 was detected in all prostate cancer cell lines and 47 frozen PCa tissues. What's more, the downregulation of DACT-2 expression could be remarkably reversed by treatment with the DNA demethylating agent 5-AZA in all PCa cell lines. Taken together, the above findings indicated that one important mechanism for transcriptional silencing of DACT-2 is hypermethylation of $\mathrm{CpG}$ sites within the DACT-2 promoter in prostate cancer. However, DACT-2 protein levels were not downregulated in 15 methylated PCa tissues. One possible explanation for this finding is that other mechanisms may also play an important role in the epigenetic silencing of DACT-2 in PCa. Thus, the detailed mechanisms underlying the downregulation of DACT-2 expression in PCa should be further investigated.

As a potent DNA methyltransferase inhibitor, 5-AZA has been used in preclinical and clinical trials to alleviate the patient's symptoms of many diseases including myelodysplastic syndrome, chronic myeloid leukemia, acute myeloid leukemia, acute lymphoid leukemia, melanoma, colorectal cancer, non-small cell lung cancer, and renal cancer ${ }^{[16-21]}$ and has recently been approved by the Food and Drug Administration for the treatment of myelodysplastic syndrome[22]. In cell culture studies, 5-AZA has been found to be useful as an inhibitor of transcripts silencing that can restore the expression of DACT-2, which was consistent of many reports concerning DACT-2 hypermethylation in other cancer[23-26]. Moreover, Zorn et al. showed that 5-AZA can delay androgen-independent disease and improve survival in the transgenic adenocarcinoma of the mouse prostate mouse model of prostate cancer[27]. An independent study also demonstrate that targeting the unique methylation pattern of androgen receptor (AR) promoter in prostate stem/progenitor cells with 5-AZA can suppress prostate tumorigenesis ${ }^{[28]}$. These results suggested that 5-AZA also has the potential as a clinical drug for prostate cancer. However, 5-AZA is broad-spectrum inhibitors of DNA methylation and may have drug side effects for some patients. Accordingly, the potential value of the application of 5-AZA as a therapeutic agent for prostate cancer should be excavated as far as possible in the future.

\section{Conclusions}

In conclusion, our research pointed out that the DACT-2 gene is a potential target for epigenetic silencing in human prostate cancer. In view of the vital function of the DACT gene family in development and carcinogenesis, the phenomenon of frequent abnormalities of epigenetic may count a great deal in the ongoing progression of prostate cancer.

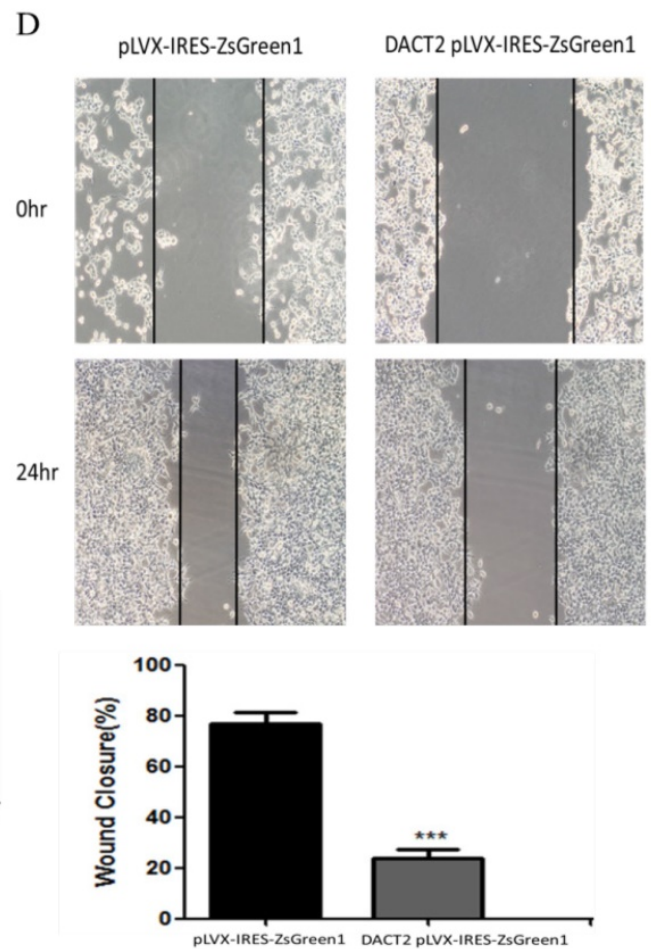

Figure 7. DACT-2 overexpression inhibited the migration and invasiveness of PC-3 cells. A, Western blot analysis of DACT-2 protein (83 kDa band) expression in PC-3 cells after transfection with DACT-2 lentiviral vector. B, Cell migration activity determined with the transwell assay. C, Cell invasion activity determined with the Matrigel invasion assay $(\times 100)$. D, Cell migration activity determined with the wound healing assay. All experiments were performed in triplicate. Data are shown as the mean \pm SD. $* * * p<0.01$ vs. $\mathrm{PLVX}-I R E S-Z s G r e e n 1$ group. 


\section{Acknowledgments}

The study was supported by the grant from the Department of Science \& Technology, Xuzhou, Jiangsu, China (No. KC16SL109, KC17089, KC17169) and the Jiangsu Provincial Commission of Health and Family Planning (No. Q2017010), the Jiang Su Provincial Medical Youth Talent(No. QNRC2016781), the Natural Science Foundation of Jiangsu Province (No.BK20160233), and the National Natural Science Foundation of China (No. 81702061).

\section{Author contributions}

LS, YL and HK contributed equally to this work. LS, YL HK and MP designed the experiments and wrote the manuscript; LS, YL, ZY and ZH performed the research and analyzed the data; CC, HK, XY and $\mathrm{LH}$. analyzed the clinical data and contributed to clinical samples; YL, WX, HL and LT did the immunohistochemical analysis and histologic assessment; LS and MP designed the study and drafted the manuscript.

\section{Competing Interests}

The authors have declared that no competing interest exists.

\section{References}

1. Siegel RL, Miller KD, Jemal A. Cancer statistics, 2016. CA Cancer J Clin. 2016; 66:7-30

2. Smith RA, Andrews K, Brooks D. et al. Cancer screening in the United States, 2016: A review of current American Cancer Society guidelines and current issues in cancer screening. CA Cancer J Clin. 2016;66:96-114

3. Bray F, Ren JS, Masuyer E. et al. Global estimates of cancer prevalence for 27 sites in the adult population in 2008. Int J Cancer. 2013;132:1133-45

4. Soerjomataram I, Lortet-Tieulent J, Parkin DM. et al. Global burden of cancer in 2008: a systematic analysis of disability-adjusted life-years in 12 world regions. Lancet. 2012;380:1840-50

5. Cui K, Li X, Du Y. et al. Chemoprevention of prostate cancer in men with high-grade prostatic intraepithelial neoplasia (HGPIN): a systematic review and adjusted indirect treatment comparison. Oncotarget. 2017:8:36674-36684

6. Lippman SM, Hong WK. Cancer prevention science and practice. Cancer Res. 2002;62:5119-25

7. Kumar NB, Pow-Sang J, Egan KM. et al. Randomized, Placebo-Controlled Trial of Green Tea Catechins for Prostate Cancer Prevention. Cancer Prev Res (Phila). 2015;8:879-87

8. Cucchiara V, Yang JC, Mirone V. et al. Epigenomic Regulation of Androgen Receptor Signaling: Potential Role in Prostate Cancer Therapy. Cancers (Basel). 2017;9:9

9. Katoh M, Katoh M. Identification and characterization of human DAPPER1 and DAPPER2 genes in silico. Int I Oncol. 2003·22.907-13

10. Wang S, Dong Y, Zhang Y. et al. DACT2 is a functional tumor suppressor through inhibiting Wnt/ $\beta$-catenin pathway and associated with poor survival in colon cancer. Oncogene. 2015;34:2575-85

11. Yu Y, Yan W, Liu X. et al. DACT2 is frequently methylated in human gastric cancer and methylation of DACT2 activated Wnt signaling. Am J Cancer Res. 2014;4:710-24

12. Zhao Z, Herman JG, Brock MV. et al. Methylation of DACT2 promotes papillary thyroid cancer metastasis by activating Wnt signaling. PLoS One. 2014;9:0112336

13. Zhang $\mathrm{M}$, Linghu $\mathrm{E}$, Zhan $\mathrm{Q}$. et al. Methylation of DACT2 accelerates esophageal cancer development by activating Wnt signaling. Oncotarget. 2016;7:17957-69

14. Friedrichs K, Gluba S, Eidtmann H. et al. Overexpression of p53 and prognosis in breast cancer. Cancer. 1993:72:3641-7

15. Cheyette BN, Waxman JS, Miller JR. et al. Dapper, a Dishevelled-associated antagonist of beta-catenin and JNK signaling, is required for notochord formation. Dev Cell. 2002;2:449-61

16. Radsak M, Platzbecker U, Schmidt CS. et al. Infectious complications in patients with myelodysplastic syndromes: A review of the literature with emphasis on patients treated with 5-azacitidine. Eur I Haematol. 2017;99:112-118

17. Yao DM, Zhou JD, Zhang YY. et al. GPX3 promoter is methylated in chronic myeloid leukemia. Int I Clin Exp Pathol. 2015;8:6450-7

18. Oka S, Ono K, Nohgawa M. Successful treatment with azacitidine for the simultaneous occurrence of multiple myeloma and acute myeloid leukemia with concomitant $\operatorname{del}(5 \mathrm{q})$ and the JAK2 V617F mutation. Ann Hematol. 2017:96:1411-1413

19. van Vlodrop IJH, Joosten SC, De Meyer T. et al. A Four-Gene Promoter Methylation Marker Panel Consisting of GREM1, NEURL, LAD1, and NEFH Predicts Survival of Clear Cell Renal Cell Cancer Patients. Clin Cancer Res. 2017;23:2006-2018

20. $\mathrm{Hu} \mathrm{YH}, \mathrm{Chen} \mathrm{Q}, \mathrm{Lu} \mathrm{YX}$. et al. Hypermethylation of NDN promotes cell proliferation by activating the Wnt signaling pathway in colorectal cancer. Oncotarget. 2017;8:46191-46203

21. Chüeh AC, Liew MS, Russell PA. et al. Promoter hypomethylation of NY-ESO-1, association with clinicopathological features and PD-L1 expression in non-small cell lung cancer. Oncotarget. 2017;8:74036-74048

22. Bhatt G, Blum W. Making the most of hypomethylating agents in myelodysplastic syndrome. Curr Opin Hematol. 2017;24:79-88

23. Liu L, Shen H, Wang Y. CRY2 is suppressed by FOXM1 mediated promoter hypermethylation in breast cancer. Biochem Biophys Res Commun. 2017;490:44-50

24. Li J, Zhang M, He T. et al. Methylation of DACT2 promotes breast cancer development by activating Wnt signaling. Sci Rep. 2017;7:3325

25. Cao D, Li D, Huang Y. et al. 5-Azacytidine promotes invadopodia formation and tumor metastasis through the upregulation of PI3K in ovarian cancer cells. Oncotarget. 2017;8:60173-60187

26. Wang X, Gao H, Ren L. et al. Demethylation of the miR-146a promoter by 5-Aza-2'-deoxycytidine correlates with delayed progression of castration-resistant prostate cancer. BMC Cancer. 2014;14:308

27. Zorn CS, Wojno KJ, McCabe MT. et al. 5-aza-2'-deoxycytidine delays androgen-independent disease and improves survival in the transgenic adenocarcinoma of the mouse prostate mouse model of prostate cancer. Clin Cancer Res. 2007:13:2136-43

28. Tian J, Lee SO, Liang L. et al. Targeting the unique methylation pattern of androgen receptor (AR) promoter in prostate stem/progenitor cells with 5-aza-2'-deoxycytidine (5-AZA) leads to suppressed prostate tumorigenesis. I Biol Chem. 2012;287:39954-66 